\section{Childhood blindness: a UK epidemiological perspective}

JS Rahi' ${ }^{1,2,3}$

\begin{abstract}
The purposes of this short paper are to introduce the reader to the aims of ophthalmic epidemiology and its relevance to clinical practice in ophthalmology and to vision science research. Summarise what is known currently about the epidemiology of severe visual impairment and blindness in childhood in the UK. Highlight the key gaps in our knowledge in this area and identify some of the most pressing challenges in terms of research.
\end{abstract}

Eye (2007) 21, 1249-1253; doi:10.1038/sj.eye.6702837

Keywords: epidemiology; childhood blindness

\section{Ophthalmic epidemiology}

Epidemiology (literally 'studies upon people ${ }^{1}$ ) has both its origins and its applications firmly rooted in clinical and public health ophthalmology.

Through ophthalmic epidemiological research we aim to

- understand the natural history and aetiology of ophthalmic disorders;

- improve the accuracy and efficiency of diagnosis;

- enhance the effectiveness of treatment and preventive strategies;

- inform the planning and provision of services

Clinical decisions and those related to service provision are, knowingly or otherwise, usually based on a 'three-part question' that incorporates the reference population (eg children under 2 years with congenital cataract), the risk factor or the intervention (eg prematurity or intraocular lens implantation), and the outcomes of interest (eg parent-reported improvement in quality of life or objective improvement in acuity and stereopsis).

Importantly, this question determines the epidemiological study design required to address it, for example, a descriptive study (eg cross-sectional prevalence study) or an analytical study (either observational, eg case-control or cohort studies or interventional, eg randomised controlled trials).

The following important issues pose particular challenges to undertaking epidemiological research on childhood visual disability.

\section{Case definition}

There is no internationally agreed definition that can be readily applied to all children with visual impairment, which limits the ability to make comparisons within and between countries, and over time, of the frequency, causes, and treatment/prevention of visual impairment. Thus, the WHO's taxonomy, ${ }^{2}$ based on acuity, has been adopted for much epidemiological research, despite the recognised difficulties of objectively measuring vision in very young children and others unable to cooperate with formal testing.

\section{Rarity}

The fact that visual impairment and blindness in childhood is uncommon poses significant methodological challenges in trying to achieve sufficiently large and representative populations of affected children to allow unbiased and meaningful study.

\section{Complex, multidisciplinary management}

For a complete picture, information must be sought from the different professionals involved in the care of visually impaired or blind children, which, in the case of the many children with additional non-ophthalmic
${ }^{1}$ Centre for Paediatric Epidemiology, Institute of Child Health, London, UK

${ }^{2}$ Department of Ophthalmology, Great Ormond Street Hospital, London, UK

${ }^{3}$ Division of Epidemiology, Institute of Ophthalmology, London, UK

Correspondence: JS Rahi, Centre for Paediatric Epidemiology, Institute of Child Health, London, UK

Tel: + 440207905 2250; Fax: +440207905 2381 E-mail: j.rahi@ich.ucl.ac.uk

Received: 4 March 2007 Accepted in revised form: 21 March 2007

Paper presented at the 36th Cambridge Ophthalmological Symposium, September 2006 
impairments or chronic disorders, adds even further layers of complexity.

\section{Long-term outcomes}

In paediatric ophthalmology, as in all paediatric disciplines, developmental issues have to be accounted for, and thus assessment of meaningful outcomes such as final visual function or educational placement requires long-term follow-up in epidemiological studies.

\section{Epidemiology of severe visual impairment or blindness} in childhood in the UK

\section{Potential sources of information}

The following are sources that one could turn to for epidemiological information about childhood visual disability in the UK, but each has specific limitations, explaining the incomplete picture that exists.

\section{Population-based prevalence studies}

Although the ideal source for robust information, studies of whole populations of children identifying those with visual impairment, such as the British national birth cohort studies, ${ }^{3,4}$ are uncommon, as they need to be very large (eg a study of 100000 children would be required in an industrialised country to identify 100-200 children with visual impairment or blindness), and thus are costly and difficult to conduct.

\section{Population-based incidence studies}

Even greater difficulties exist in conducting incidence studies, explaining the greater paucity of incidence data.

Special needs/disability registers, surveys, and surveillance Specific studies and/or surveillance systems ${ }^{5}$ or registers of childhood disability can provide information about visual impairment, but it is important to recognise the potential for bias as certain visually impaired children may be overrepresented in these sources, for example, those with multiple impairment.

Visual impairment (blindness and partial sight) registers These have existed for many years in the UK but certification, which is voluntary and not a prerequisite for accessing special educational or social services, is recognised to be incomplete and biased, reflecting differences in both parental preferences and professionals' practices regarding registration of eligible children. ${ }^{6}$

\section{Visual impairment teams}

Increasingly children in industrialised settings are evaluated by multidisciplinary teams, and if these serve geographically defined populations then useful information about visual impairment can be derived.

\section{Service-linked databases}

There are opportunities for gathering epidemiologically related information from voluntary sector and other organisations that provide information and support to families of visually impaired children, for example, VISKIDS, a new initiative (www.viscotland.org.uk) in Scotland. It is important to recognise the potential for bias without careful assessment of the information, as such sources rely on self-notification by parents of their children rather the application of a standardised definition based on acuity. Thus, for example, children with visual processing problems or unilateral eye disease which had a functional impact on education would be included alongside visually impaired children with bilateral eye disease.

Disorder-specific ophthalmic surveillance schemes Research on uncommon ophthalmic conditions in children can be undertaken using a range of specific population-based surveillance schemes, for example, those for congenital anomalies (eg for study of anophthalmia or microphthalmia) or adverse drug reactions (eg for study of visual loss with vigabatrin) although under-ascertainment in such work is recognised. The national active surveillance scheme, comprising all senior ophthalmologists in the United Kingdom (the British Ophthalmological Surveillance Unit $^{7}$ ), provides an important mechanism model for ophthalmic epidemiological research on uncommon disorders. It has facilitated the study of a number of paediatric ophthalmic disorders, including retinopathy of prematurity, congenital glaucoma, amblyopia, hereditary retinal diseases, and congenital anomalies, ${ }^{8,9}$ as well as the incidence study of severe visual impairment and blindness in childhood whose findings are summarised below.

\section{Summary of current data on severe visual impairment and blindness in the UK}

In the light of the limitations of existing available sources, the first population-based study of severe visual impairment and blindness in childhood was undertaken in the UK in 2000, through a novel collaborative clinical research group. Findings have been reported in detail previously, ${ }^{10}$ but key data are summarised here.

\section{Demographics}

Of 439 children who were newly diagnosed as being severely visually impaired or blind (SVI/BL) in 2000, $54 \%$ were boys, $72 \%$ were white, $24 \%$ weighed less than 
$2500 \mathrm{~g}$ at birth, and $40 \%$ were in the most deprived national quintile of the Carstairs index. Seventy-seven per cent $(336 / 438)$ of all these children had associated non-ophthalmic disorders and/or impairments ('SVI/BL plus'). Those of low birth weight $(<2500 \mathrm{~g})$ were more likely than those of normal birth weight to have SVI/BL plus (OR 5.9, 95\% CI 2.4-15.8, $P<0.001$ ), but no association was found with sex, ethnic group, or deprivation score.
Incidence

The total annual age-specific incidence was highest in the first year of life, being $4.0(3.6-4.5)$ per 10000 , with a cumulative incidence by 16 years of 5.9 (5.3-6.5) per 10000 . The rate of 'SVI/BL plus' is higher than that of isolated SVI/BL at all ages. Incidence was higher among all ethnic minority groups and, in the first year of life, varied inversely with birth weight. Incidence did not vary by sex or country of residence.

Table 1 Classification of the causes of childhood visual impairment or blindness, according to the anatomical site(s) affected, and the aetiological factors by their timing of action

\begin{tabular}{|c|c|}
\hline Anatomical site(s) affected & Aetiological factor(s) by timing of action \\
\hline Whole globe and anterior segment & Prenatal \\
\hline Microphthalmia/anophthalmia & Hereditary \\
\hline Anterior segment dysgenesis & Autosomal recessive, autosomal dominant, $\mathrm{X}$-linked \\
\hline Coloboma-multiple sites & Chromosomal \\
\hline Others & Hypoxia/ischaemia \\
\hline Glaucoma & Infection \\
\hline Primary & Prenatal drug \\
\hline Secondary & Others \\
\hline Cornea & Presumed prenatal but factor unknown \\
\hline Sclerocornea & \\
\hline Keratomalacia & Perinatal + neonatal \\
\hline Other corneal scar & Hypoxia/ischaemia \\
\hline Lens & Infection \\
\hline Cataract & Non-accidental injury \\
\hline Aphakia & Others \\
\hline Subluxed & Presumed peri/neonatal but factor unknown \\
\hline \multicolumn{2}{|r|}{ 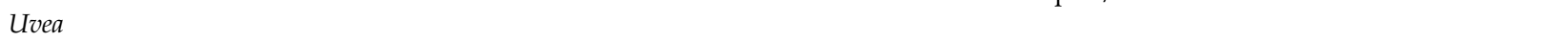 } \\
\hline Anidiria & Childhood \\
\hline Uveitis & Tumour \\
\hline Coloboma-single site & Nutritional \\
\hline Retina & Infection \\
\hline Retinopathy of prematurity & Hydrocephalus/increased cranial pressure \\
\hline Retinal and macular dystrophies & Hypoxia / ischaemia \\
\hline Oculo-cutaneous albinism & Non-accidental injury \\
\hline Retinitis/neuroretinitis & Accidental injury \\
\hline Retinal detachment & Specific systemic disorders \\
\hline Retinoblastoma & Presumed childhood but factor unknown \\
\hline \multicolumn{2}{|r|}{ ( } \\
\hline Optic nerve & Undetermined timing of insult and factors unknown \\
\hline \multicolumn{2}{|l|}{ Hypoplasia } \\
\hline \multicolumn{2}{|l|}{ Atrophy (primary or secondary) } \\
\hline \multicolumn{2}{|l|}{ Neuritis/neuropathy } \\
\hline \multicolumn{2}{|l|}{ Others } \\
\hline \multicolumn{2}{|l|}{ Cerebral/visual pathways } \\
\hline \multicolumn{2}{|l|}{ Neuro-degenerative disorders } \\
\hline \multicolumn{2}{|l|}{ Hypoxic/ischaemic encephalopathy } \\
\hline \multicolumn{2}{|l|}{ Non-accidental injury } \\
\hline \multicolumn{2}{|l|}{ Infection } \\
\hline \multicolumn{2}{|l|}{ Structural abnormalities } \\
\hline \multicolumn{2}{|l|}{ Tumour } \\
\hline \multicolumn{2}{|l|}{ Other } \\
\hline \multicolumn{2}{|l|}{ Other } \\
\hline \multicolumn{2}{|l|}{ Idiopathic nystagmus } \\
\hline High refractive error & \\
\hline
\end{tabular}

Modified from the WHO/PBL Eye Examination Record. ${ }^{11}$ 
Ten per cent (44/439) of all children died within a year of diagnosis, $77 \%$ (34) in the first year of life. Thus, infant mortality among SVI/BL was 119.3 per 1000 (95\% CI 81.4-156.4). All those who died had additional nonophthalmic disorders, which were the cause of death, and most had ophthalmic diseases affecting the visual pathways or cortex and/or optic nerve.

\section{Anatomical site(s) affected}

A single anatomical site was reported to be affected in $77 \%$ of all children, with multiple main sites in the remaining children. Cerebral visual impairment (CVI), retinal disorders, and disorders of the optic nerve were the main (or sole) disorders, affecting 48, 29, and $28 \%$ of all children, respectively (Table 1 ).

CVI and disorders of the optic nerve (particularly optic atrophy) were more common among those with SVI/BL plus than those with isolated SVI/BL. Conversely, retinal disorders (particularly retinal dystrophies and albinism) and glaucoma were relatively more important in those with isolated visual loss.

Children of low birth weight $(<2500 \mathrm{~g})$ were significantly more likely to have CVI than those of normal birth weight $(60 \%, 58$ vs $43 \%$, 132, respectively, 95\% CI for difference $6-29 \%, P=0.005)$. There were no significant variations in the anatomical pattern according to sex.

Timing of insult(s) and aetiological factors (Table 1) The timing of the insult resulting in SVI/BL was ascribed to a single category in $95 \%$ of all children. Prenatal factors (acting at conception or during pregnancy) predominated overall, and were relatively more important in those with isolated SVI/BL. Conversely, perinatal/neonatal and childhood factors were significantly more important in those with additional non-ophthalmic impairments (SVI/BL plus).

Prenatal factors affected $49 \%$ of children weighing $<2500$ g compared with $67 \%$ of those $2500 \mathrm{~g}$ or more at birth (95\% CI for difference 18-39\%, P<0.001). Conversely, $39 \%$ of low, compared with $10 \%$ (30) of normal birth weight children had perinatal/neonatal factors $(95 \%$ CI 7-29\%, P<0.001). Hereditary disorders (classified within the prenatal category) affected $46 \%$ (32) of south Asian (Indian, Pakistani, or Bangladeshi) children compared with $29 \%$ (86) of white, $33 \%$ (6) of black and 33\% (9) of other ethnic groups (95\% CI for difference between south Asian and white 3-28\%, $P=0.01)$. There were no significant differences according to sex or deprivation score, although the latter is not an individual level measure and the overrepresentation of children from more deprived groups may have limited the ability to detect true differences.
Avoidable severe visual impairment and blindness With currently available measures in the UK, SVI/BL (although not necessarily visual loss per se) was potentially avoidable in up to $25 \%$ of children. The proportion of preventable or treatable disorders was similar for those with SVI/BL plus and those with isolated SVI/BL. However, the ophthalmic disorders differed, with those in the isolated group, such as cataract or glaucoma, being more straightforward to treat.

\section{Summary and implications}

Blindness in children in the UK should be viewed within the broader context of disability and chronic disease in childhood and the important influences on the health of children of birth weight, socio-economic deprivation, and ethnicity. Novel interventions to address the currently largely insurmountable burden of blinding eye disease are required and their development will necessitate integration of clinical, epidemiological, and basic scientific research.

\section{Current gaps in our knowledge}

(1) There is limited information available about the frequency and causes of childhood visual impairment in childhood (vs blindness, as described above). The incidence of this level of impairment is likely to be much higher and the pattern of causes (disorders and aetiological factors) is also likely to differ substantially. This paucity is a particularly important gap in the evidence base that underpins clinical practice, service provision, and research.

(2) There is limited information available about the long-term outcomes and natural history of visually impairing disorders of childhood. There has been remarkably limited research investigating the impact in later life on social outcomes (eg education and occupation), general and mental health, and quality of life of affected individuals and their families. This dearth of information makes it difficult to assess and interpret the relative importance of vision in relation to health and disease in childhood, for example, in international burden of disease studies.

(3) There is limited information available on the costeffectiveness of different treatment and preventive strategies for visually impairing disorders of childhood. There have been few health economic studies of interventions for visual impairment, partly because of the paucity of natural history data that are required for economic modelling. The paucity of information in this area has important implications for debates about resource allocation. 


\section{Key challenges for future epidemiological research}

Much further epidemiological research is necessary to build a complete picture of childhood visual impairment in the UK. This work would be greatly facilitated by advances in three key areas:

1. The development and application of an internationally agreed system of classification of childhood visual disability, which is applicable to children of different ages and which may allow consideration of other visual parameters such as near acuity, visual fields, binocularity, and contrast sensitivity.

2. The development of a robust and responsive system for monitoring the frequency and causes of visually impairing diseases of childhood.

3. The establishment of large-scale interdisciplinary collaborations (eg national clinical research networks) to provide the infrastructure necessary for effective research.

\section{References}

1 Last JM. A Dictionary of Epidemiology, 2nd edn. Oxford University Press: Oxford, 1988.

2 World Health Organisation (WHO). International Statistical Classification of Diseases and Health Related Problems, 10th revision. World Health Organisation: Geneva, 1922.
3 Peckham C, Pearson R. Preliminary findings at the age of 16 years in children in National Child Development Study (1958 cohort). Public Health 1976; 90: 271-280.

4 Stewart-Brown S, Haslum MN. Partial sight and blindness in children of the 1970 birth cohort at 10 years of age. J Epidemiol Community Health 1988; 42: 17-23.

5 Mervis CA, Yeargin Allsopp M, Winter S, Boyle C. Aetiology of childhood vision impairment, metropolitan Atlanta, 1991-1993. Paediatr Perinat Epidemiol 2000; 14: 70-77.

6 Evans J. Causes of Blindness and Partial Sight in England and Wales 1990-91. Studies on Medical and Population Subjects No 5. HMSO: London, 1995.

7 Foot B, Stanford MR, Rahi JS, Thompson JR, on behalf of the British Ophthalmological Surveillance Unit. The British Ophthalmological Surveillance Unit: an evaluation of the first three years. Eye 2003; 17: 9-16.

8 Haines L, Fielder AR, Baker H, Wilkinson AR. UK population based study of severe retinopathy of prematurity: screening, treatment and outcome. Arch Dis Child Fetal Neonatal Ed 2005; 90: F240-F244.

9 Rahi JS, Logan S, Timms C, Russell-Eggitt I, Taylor DSI. Risk, causes, and outcomes of visual impairment after loss of vision in the non-amblyopic eye: a population-based study. Lancet 2002; 360: 597-602.

10 Rahi JS, Cable N, on behalf of the British Childhood Visual Impairment Study Group (BCVISG). Severe visual impairment and blindness in children in the UK. Lancet 2003; 362: 1359-1365.

11 Gilbert C, Foster A, Negrel A-D, Thylefors B. Childhood blindness: a new form for recording causes of visual loss in children. Bull World Health Organ 1993; 71: 485-489. 